\title{
Presumed Encephalitis With a Reversible Splenial Lesion
}

\author{
Nora Moron Cabrera ${ }^{\mathrm{a}}$, Evelyn Calderon Martinez ${ }^{\mathrm{b}, \mathrm{d}}$, \\ Maria Moron Cabrera ${ }^{\mathrm{c}}$, Lelia Romero ${ }^{\mathrm{c}}$
}

\begin{abstract}
Mild encephalitis/encephalopathy with a reversible splenial lesion (MERS) is a clinicoradiologic syndrome which consists of a transient lesion usually located in the midline of the corpus callosum that shows restricted diffusion. The exact pathophysiology of this entity is still unknown. We present the case of a 9-year-old boy with a history of 3 days of headache and vomiting associated with an episode of syncope and mild hyponatremia. Magnetic resonance imaging (MRI) findings included a focal lesion with well-defined margins on the splenium of the corpus callosum with hyperintensity on $\mathrm{T} 2$ and diffusion-weighted images. The patient underwent full recovery, and a follow-up MRI 5 months later showed no signs of the initial lesion. In conclusion, MERS is part of the spectrum of cytotoxic lesions of the corpus callosum, which must be recognized as secondary to a several causes, to avoid an erroneous diagnosis and treatment of the patient. It should be emphasized the importance of continuing research on this type of process to clarify its pathophysiology as well as the long-term effects.
\end{abstract}

Keywords: Encephalitis; Encephalopathy; MERS; MRI

\section{Introduction}

Mild encephalitis/encephalopathy with a reversible splenial lesion (MERS) is a relatively new clinicoradiologic syndrome that belongs to the group of transient lesions of the corpus callosum. The mechanism of this injury is still unknown; however, it is believed to be due to a focal cytotoxic edema of the corpus callosum in response to an initial injury that may correspond to multiple causes, including some bacterial and viral agents [1]. Given that the corpus callosum is the biggest fiber bundle with multiple connections with motor and sensory ar-

Manuscript submitted September 2, 2020, accepted October 26, 2020

Published online November 27, 2020

aUniversity of San Martin de Porres, Lima, Santa Anita 15011, Peru

bUniversity of Guayaquil, Guayaquil 090613, Ecuador

'Department of Radiology, Edgardo Rebagliati Martins National Hospital, Jesus Maria 15072, Peru

${ }^{\mathrm{d} C}$ Corresponding Author: Evelyn Calderon Martinez, University of Guayaquil, Avenida 10 NO, Guayaquil 090613, Ecuador.

Email: ejcalderonm@gmail.com

doi: https://doi.org/10.14740/ijcp405 eas, the manifestations of MERS can include seizures, mental status alteration, disorder of motor control, spatial orientation, vision (including transient blindness), hearing, and languagerelated behaviors [2]. Patients can also present fever, cough, vomiting and/or diarrhea. The magnetic resonance imaging (MRI) typically shows restricted diffusion in the splenium of the corpus callosum. Clinical outcomes are excellent; in most cases a complete resolution of symptoms is seen within a week with no long-term sequelae [3].

Although the majority of MERS cases have been reported in East Asia and European countries, here we present a Peruvian pediatric patient with typical clinical and radiologic features of MERS after presumed viral encephalitis.

\section{Case Report}

A 9-year-old male patient presented with headache and vomiting for 3 days that initially responded to analgesics and was discharged. Two days later, the symptoms reappeared in greater magnitude and were associated with profuse sweating and one episode of syncope, did not report ill contacts. The patient was admitted for further investigation. On admission the patient had normal vital signs, his weight was $40 \mathrm{~kg}$, and his height was $1.5 \mathrm{~m}$. On physical examination the patient had irritability without other neurological alteration; cranial nerves 2 - 12 were tested and were grossly intact, deep tendon reflexes of upper and lower extremities were symmetrical; gait was normal. No sensory or motor alterations were noted. The remainder of the physical examination was unremarkable. Laboratory results on admission showed hyponatremia (sodium $115 \mathrm{mEq} / \mathrm{L}$ ) which was corrected with oral salt and water restriction.

The patient was born at 36-week gestation by cesarean with no perinatal complications, his vaccinations were up to date, and past medical history included only a left arm fracture corrected with surgery.

The computed tomography (CT) scan on admission did not show alterations. A MRI performed 2 days later showed ill-defined, symmetrical, and hyperintense lesions in the deep white matter, predominantly in the centrum semiovale and a well-demarcated ovoid lesion in midline of the splenium of the corpus callosum (white arrows in Fig. $1 \mathrm{a}, \mathrm{b}$ ), hypointense on T1 and hyperintense on T2 (Fig. 2), that shows a marked reduced diffusion (apparent diffusion coefficient (ADC) map) and does not show enhancement with contrast (Fig. $3 \mathrm{a}, \mathrm{b}$ ).

A presumptive diagnosis of viral encephalitis was made; 


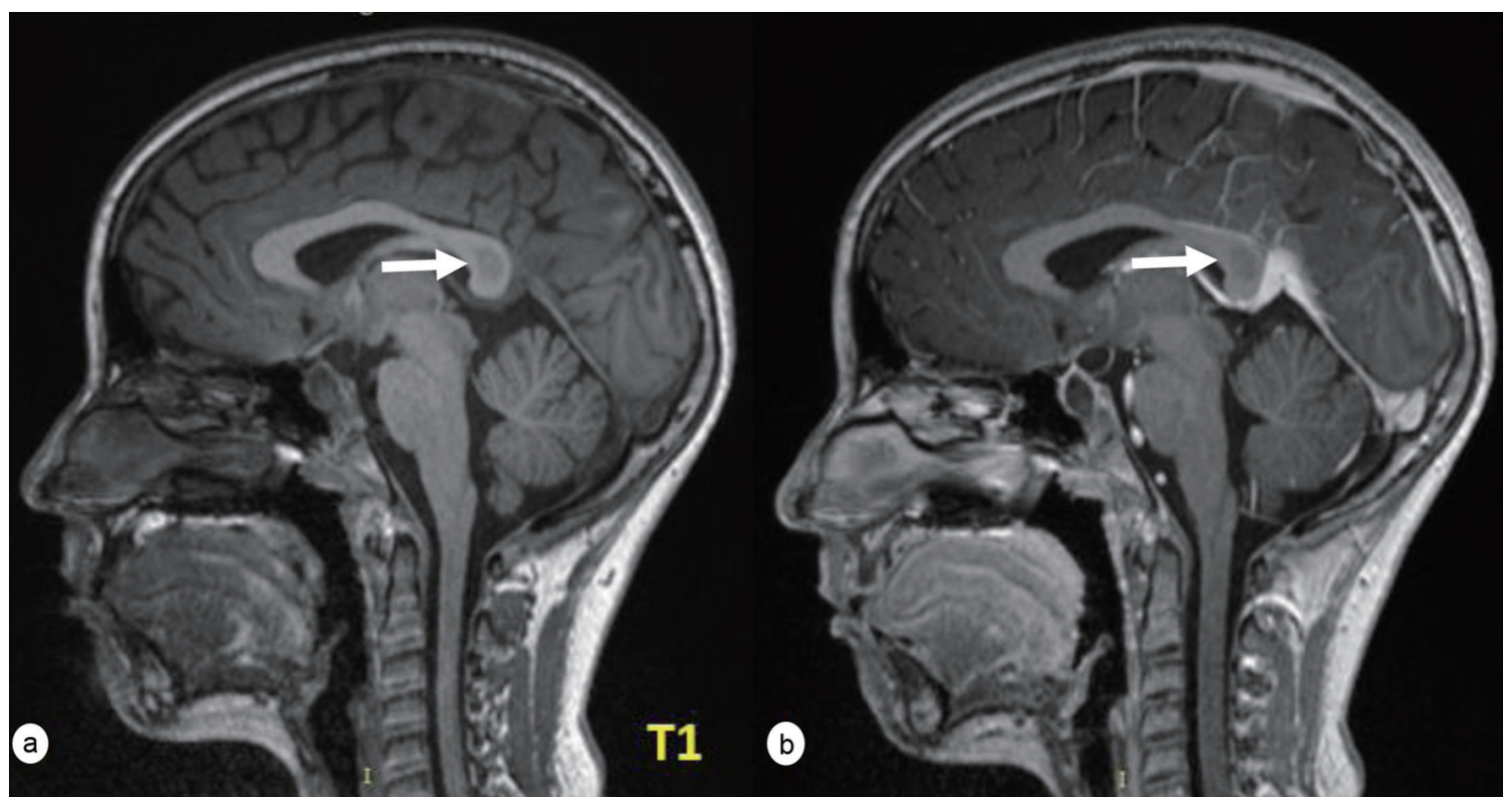

Figure 1. Well-demarcated ovoid lesion (a) in the midline of the splenium of the corpus callosum (white arrows) that does not show uptake after the intravenous administration of paramagnetic contrast (b).

however, the etiological agent could not be identified. The symptoms improved with supportive care and the patient was discharged after 12 days of hospitalization. A control MRI was

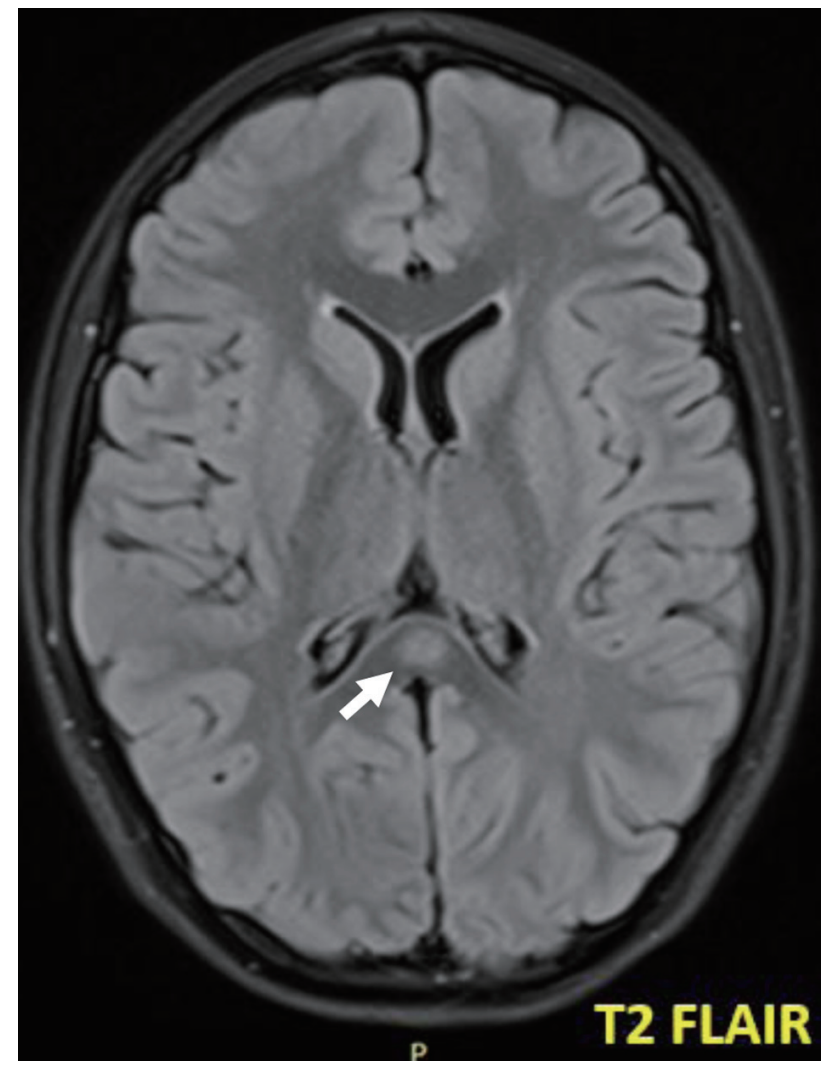

Figure 2. The same lesion, seen as hyperintense in FLAIR (white arrow). FLAIR: fluid-attenuated inversion recovery. performed 5 months later that demonstrated complete resolution of the previously seen lesions (Fig. 4). Currently, he refers to having returned to his daily activities without limitations or suggestive signs of neurological sequelae, his overall development has been normal, and he has met all developmental milestones.

\section{Discussion}

Although the majority of MERS cases have been reported in East Asia and European countries, here we present a Peruvian pediatric patient with typical clinical and radiologic features of MERS after presumed viral encephalitis. A 9-year-old boy with a history of 3 days of headache and vomiting associated with an episode of syncope and mild hyponatremia. On admission vital signs were within normal limits and physical examination was remarkable for irritability only with any other neurologic abnormality. MRI findings included a focal lesion with well-defined margins on the splenium of the corpus callosum with hyperintensity on T2 and diffusion-weighted images. The patient underwent full recovery with no neurological sequelae, and follow-up MRI 5 months later showed no signs of the initial lesion.

The exact etiopathogenesis of MERS is not clear yet, but it is believed that the lesions seen in this entity are caused by several inflammatory cytokines (specially interleukin 1 (IL-1) and IL-6) released in response to an initial injury, which causes the recruitment of $\mathrm{T}$ cells, weakening of the blood-brain barrier, tumor necrosis factor- $\alpha(\mathrm{TNF} \alpha)$ production and astrocyte activation. A result there is a large cytotoxic edema mainly due to increased extracellular glutamate, especially in the splenium of the corpus callosum where a large number of glutamate receptors are found [4]. It is also mentioned that hyponatremia could play a role in the physiopathology of MERS as seen in 


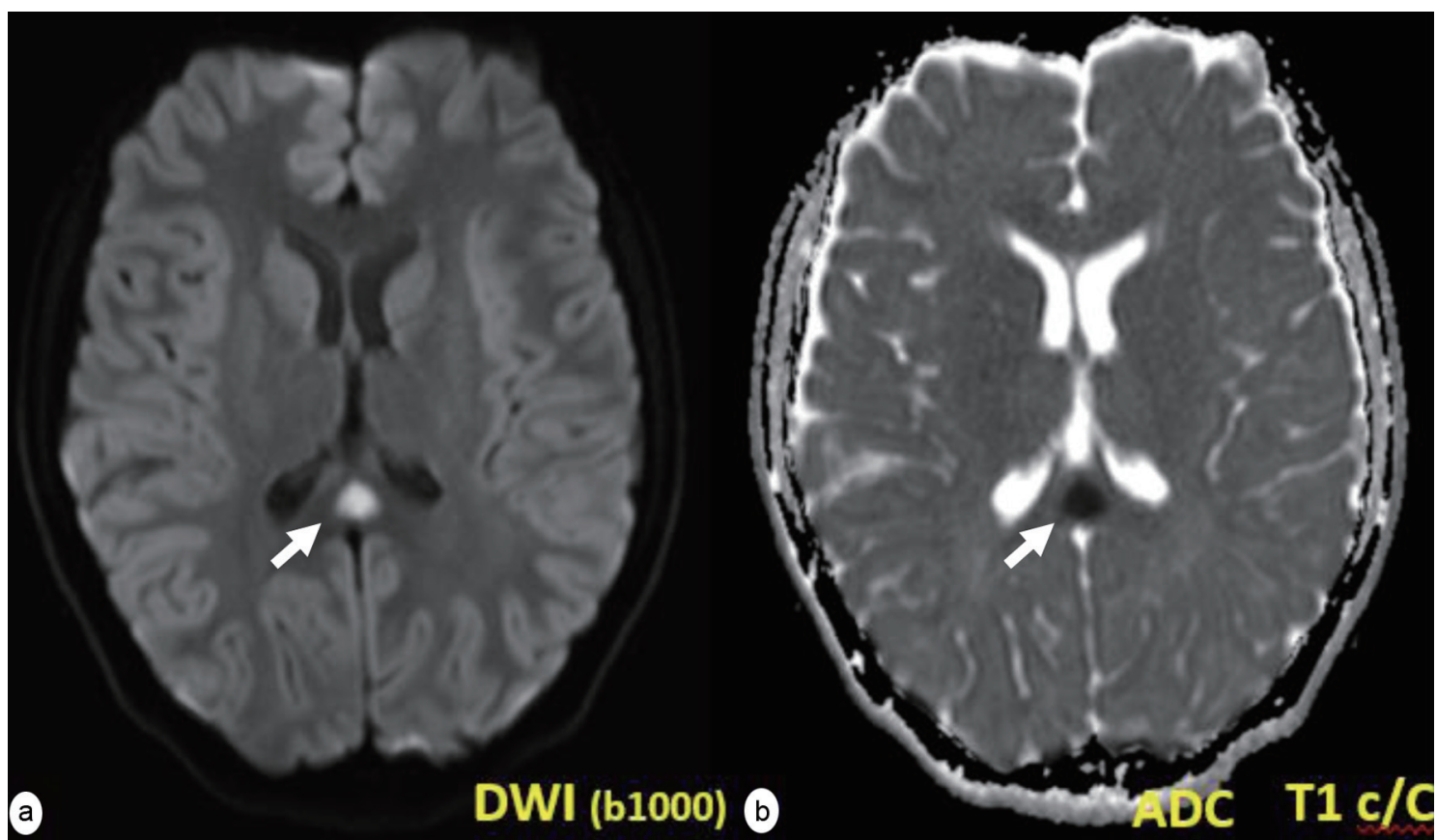

Figure 3. The same lesion that shows a marked reduced diffusion (white arrows). DWl: diffusion-weighted imaging; ADC: apparent diffusion coefficient.

the reported case [5].

Although this case was caused by presumed viral encephalitis, these types of lesions can be triggered by other factors such as some bacterial and viral agents like rotavirus, adenovirus, influenza, dengue virus, mumps virus, Mycoplasma pneu-

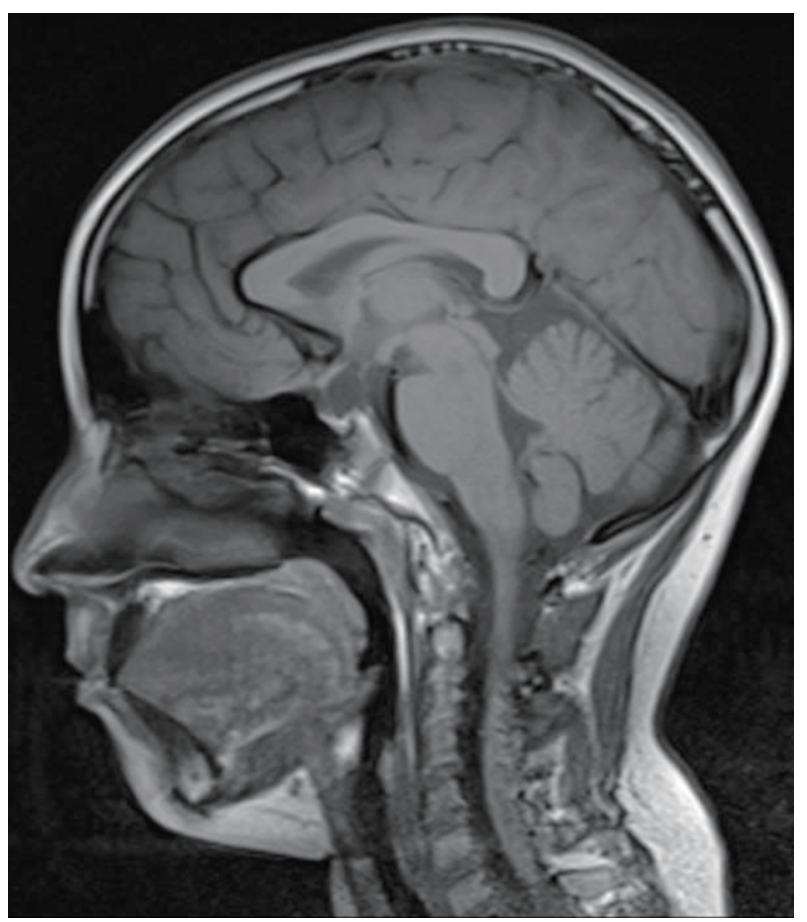

Figure 4. Sagittal T1-weighted control performed 5 months later with complete resolution of the previous lesion. moniae, Streptococcus pneumoniae [1], drugs, central nervous system (CNS) and gastrointestinal infections, hemolytic uremic syndrome, subarachnoid hemorrhage, metabolic disorders, trauma, among others [6-8]. The clinical presentation varies depending on the cause of the initial injury. Treatment of the underlying cause is enough to reverse the lesions [8].

MRI characteristics include marked restriction to diffusion with three patterns: 1) a rounded lesion delimited in the center of the splenium; 2) a lesion extending laterally through the callus fibers towards the white matter; or 3 ) a predominantly posterior lesion extending into the anterior portion of the corpus callosum. The complete resolution of the lesions has been described within a range of 3 days to 1 year after diagnosis [9].

Differential diagnoses include ischemic infarction due to occlusion of the distal branches of the anterior cerebral artery, tumor such as lymphoma and glioblastoma, however these tend to be more aggressive. It is also important to rule out the presence of multiple sclerosis, disseminated acute encephalomyelitis, which is differentiated by a generally asymmetric presentation. Other diseases that could simulate this process are MarchiafavaBignami, pontine myelinolysis, among others [10].

In conclusion, MERS is part of the spectrum of cytotoxic lesions of the corpus callosum, which must be recognized as secondary to a several causes, to avoid an erroneous diagnosis and treatment of the patient. It should be emphasized the importance of continuing research on this type of process to clarify its pathophysiology as well as the long-term effects.

\section{Acknowledgments}

None to declare. 


\section{Financial Disclosure}

None to declare.

\section{Conflict of Interest}

None to declare.

\section{Informed Consent}

Not applicable.

\section{Author Contributions}

Maria Moron Cabrera performed the images, made the radiologic diagnosis, analyzed the results, and collected the patient's data. Evelyn Calderon Martinez, Nora Moron Cabrera, and Lelia Romero reviewed the pertinent literature and drafted the paper. Evelyn Calderon Martinez and Nora Moron Cabrera communicated with potential journals.

\section{Data Availability}

The authors declare that data supporting the findings of this study are available within the article.

\section{References}

1. Yildiz AE, Maras Genc H, Gurkas E, Akmaz Unlu H, Oncel IH, Guven A. Mild encephalitis/encephalopathy with a reversible splenial lesion in children. Diagn Interv Radiol. 2018;24(2):108-112.

2. Jea A, Vachhrajani S, Widjaja E, Nilsson D, Raybaud C, Shroff M, Rutka JT. Corpus callosotomy in children and the disconnection syndromes: a review. Childs Nerv Syst. 2008;24(6):685-692.

3. Chow C, Ling S. Mild encephalopathy with reversible splenial lesion in children. Proceedings of Singapore Healthcare. 2012;21(4):293-296.

4. Starkey J, Kobayashi N, Numaguchi Y, Moritani T. Cytotoxic lesions of the corpus callosum that show restricted diffusion: mechanisms, causes, and manifestations. Radiographics. 2017;37(2):562-576.

5. Takanashi J, Tada H, Maeda M, Suzuki M, Terada H, Barkovich AJ. Encephalopathy with a reversible splenial lesion is associated with hyponatremia. Brain Dev. 2009;31(3):217-220.

6. Miranda J, Pereira I, Nunes J, Santos F. Encefalitis/encefalopatia leve con lesion reversible del esplenio del cuerpo calloso asociada a pielonefritis aguda; a proposito de un caso clinico. Neurologia. 2018.

7. Takanashi J, Shiihara T, Hasegawa T, Takayanagi M, Hara M, Okumura A, Mizuguchi M. Clinically mild encephalitis with a reversible splenial lesion (MERS) after mumps vaccination. J Neurol Sci. 2015;349(1-2):226-228.

8. Singh P, Gogoi D, Vyas S, Khandelwal N. Transient splenial lesion: Further experience with two cases. Indian J Radiol Imaging. 2010;20(4):254-257.

9. Malhotra HS, Garg RK, Vidhate MR, Sharma PK. Boomerang sign: Clinical significance of transient lesion in splenium of corpus callosum. Ann Indian Acad Neurol. 2012;15(2):151-157.

10. Yuan J, Yang S, Wang S, Qin W, Yang L, Hu W. Mild encephalitis/encephalopathy with reversible splenial lesion (MERS) in adults-a case report and literature review. BMC Neurol. 2017;17(1):103. 